\title{
Biofilm Deformation in Response to Fluid Flow in Capillaries
}

\author{
Garret D. Vo, ${ }^{1,2}$ Jeffrey Heys ${ }^{2,3}$ \\ ${ }^{1}$ Mechanical Engineering, Montana State University, Bozeman, Montana 59717 \\ ${ }^{2}$ Center for Biofilm Engineering, Montana State University, Bozeman, Montana; \\ telephone: 406-994-7902, fax: 406-994-5308; e-mail: jeff.heys@gmail.com \\ ${ }^{3}$ Chemical and Biological Engineering, Montana State University, Bozeman, Montana 59717 \\ Received 15 November 2010; revision received 5 March 2011; accepted 10 March 2011 \\ Published online 17 March 2011 in Wiley Online Library (wileyonlinelibrary.com). DOI 10.1002/bit.23139
}

ABSTRACT: Biofilms are complex mixtures of microorganisms and extracellular matrix that exist on many wetted surfaces. Recently, magnetic resonance microscopy has been used to measure fluid velocities near biofilms that are attached to the walls of capillary channels. These velocity measurements showed unexpectedly high secondary velocities (i.e., high velocity magnitudes perpendicular to the direction of bulk flow and perpendicular to the surface that the biofilm is attached), and the presence of high secondary velocities near a biofilm could increase the delivery of substrates to the biofilm. A mathematical model, based on the immersed boundary method, is used here to examine the physical interaction between a biofilm and a moving fluid in a capillary and to analyze possible factors that may contribute to the elevated secondary velocities observed experimentally. The simulation predicts the formation of a recirculation downstream of a biofilm, and this recirculation deforms and lifts the biofilm upward from the surface to which the biofilm is attached. Changing the mechanical properties (i.e., stiffness) of the biofilm impacts both the lifting of the biofilm and the magnitude of the secondary velocities. The maximum lifting of the biofilm occurs when the biofilm properties are similar to previous experimental measurements, which indicates that the mechanical properties of the biofilm may be tuned for the generation of maximum secondary velocity magnitude and transport of substrates to the biofilm.

Biotechnol. Bioeng. 2011;108: 1893-1899.

(C) 2011 Wiley Periodicals, Inc.

KEYWORDS: biofilm; immersed boundary; secondary flow; mass transport

\section{Introduction}

The traditional view of a biofilm is as a colony of cells and extracellular polymeric substance (EPS) attached to a

Correspondence to: J. Heys

Contract grant sponsor: Flight Attendant Medical Research Institute

Contract grant sponsor: NSF

Contract grant number: CMMI-0849433; DMS-0811275 surface with either stagnate fluid or fluid flow tangential to the biofilm surface (Characklis, 1981; Costerton et al., 1995). A common laboratory setting for the study of biofilms is using a square, glass capillary, and growing biofilm colonies on the surface of the glass (Gjersing et al., 2005; Rani et al., 2005; Stewart et al., 2007). This experimental setting allows substrates such as oxygen and glucose to be delivered to the biofilm by flowing fluid through the capillary, and the biofilm may be observed through the glass walls using a microscope. The fluid flow is assumed to be primarily tangential to the biofilm and the surface to which the biofilm is attached, and substrate transport to the biofilm occurs primarily though diffusion to the biofilm surface through the concentration boundary layer and into the biofilm interior (Chambless and Stewart, 2007; Klapper and Dockery, 2010; Picioreanu et al., 2000).

In cases where the flow is assumed to be tangential to the attached surface near the biofilm, a classical mass transfer model based on the bulk tangential velocity is used (Gjersing et al., 2005; Lewandowski and Beyenal, 2007; Lewandowski et al., 1995). For example, in capillary flow experiments, the mass transfer to the biofilm is related to the axial velocity using an empirical mass transfer coefficient proportional to power law scaling of the Schmidt and Reynolds numbers (Bird et al., 2002). In other cases, where the roughness of the biofilm is accounted for in the model, the velocity is still assumed to be fully in the axial direction along the center of the glass capillary (Picioreanu et al., 2000). In biofilm experiments, it is common to refer to the axial or bulk velocity component as the primary velocity. The magnitude of other two velocity components, one of which is perpendicular to the surface to which the biofilm is attached, are called the secondary velocities. It has traditionally been assumed that secondary velocities are negligible at a distance $H$ away from the biofilm, where $H$ is the thickness of the biofilm.

The importance and large magnitude of secondary velocities was demonstrated recently using magnetic 
resonance microscopy (MRM) in two separate studies (Hornemann et al., 2009; Wagner et al., 2010). Hornemann et al. began by seeding Staphylococcus epidermidis cells onto the walls of a square glass capillary for a 4 -h period without flow. Then, the biofilm was grown for $48-96 \mathrm{~h}$ while being fed nutrient at a fixed flow rate of $16 \mathrm{~mL} / \mathrm{s}(\mathrm{Re}=8-33)$. After the biofilm was established on the walls of the capillary, it was transferred into the MRM instrument and a constant flow rate though the capillary was maintained during the experiment. The flow rate and Reynolds number through the capillary depended on the cross-sectional size: $\mathrm{Re}=$ $110 \pm 3.8$ for the $2 \mathrm{~mm}$ capillary, $\mathrm{Re}=430 \pm 30$ for the $0.9 \mathrm{~mm}$ capillary, and $\mathrm{Re}=562 \pm 172$ for the $0.5 \mathrm{~mm}$ capillary. The thickness of the biofilm was relatively independent of the capillary size, and the growth protocol for the biofilm was design to generate biofilms of approximately $100 \mu \mathrm{m}$ in thickness or $5-20 \%$ of the capillary thickness.

The velocity profiles through the capillaries measured using MRM showed peak velocities along the mid-section and no flow at the sides, as expected. Also, clean capillaries without any biofilm formation showed no secondary velocities, which is also expected under steady flow conditions. For the capillaries with a biofilm on a wall, significant secondary velocities were measured, and they had a significant magnitude well above the surface of biofilm. For example, along the centerline of the $0.9 \mathrm{~mm}$ capillary (approximately $350 \mu \mathrm{m}$ above the capillary wall, or 200$250 \mu \mathrm{m}$ above the biofilm), the secondary flow velocity was approximately $20 \%$ of the maximum primary (axial) flow. In general, the ratio of maximum secondary flow to maximum axial flow depended greatly on the size of the capillary: $0.049 \pm 0.001$ for the $0.5 \mathrm{~mm}$ capillary, $0.20 \pm$ 0.003 for the $0.9 \mathrm{~mm}$ capillary, and $0.12 \pm 0.028$ for the $2 \mathrm{~mm}$ capillary.

Previously, we developed a mathematical model to study the physical interaction between a biofilm and a moving fluid (Vo et al., 2010). The model was based on the immersed boundary method, and it was validated by comparing the model predictions of biofilm deformation magnitude and deformation time-scales with experimental measurements. The objective here is to use an advanced and updated version of this model to simulate the physical response of a biofilm to moving fluid in a square capillary consistent with the experiments of Hornemann et al. (2009). The goal is to answer a number of fundamental questions raised by those experimental results. What mechanism is responsible for the generation of the unexpectedly large secondary velocities? How do different parameters, such as the ratio of biofilm thickness to capillary size or the Reynolds number of the flow, impact the magnitude of the secondary velocities? And, most interesting, how do the mechanical properties (i.e., stiffness) of the biofilm impact the secondary velocities and is there any evidence that the mechanical properties are tuned to maximize secondary velocities for the delivery of substrates to the biofilm?

\section{Methods}

The physical interaction between a biofilm and water is modeled here using an immersed boundary algorithm (McQueen and Peskin, 2000; Peskin, 1977; Zhu and Peskin, 2002) that is similar to the algorithm that was developed and experimentally validated for biofilms in Vo et al. (2010). Here, we briefly review the model equations and described the differences between the current algorithm and that in Vo et al. (2010).

The equations of motion for a three-dimensional Newtonian, incompressible fluid are:

$$
\begin{gathered}
\frac{\partial v}{\partial t}+v \cdot \nabla v=-\nabla p+\frac{1}{\operatorname{Re}} \nabla^{2} v+f \\
\nabla \cdot v=0
\end{gathered}
$$

where $v$ and $p$ are the velocity and pressure of the fluid, respectively, and $R e$ is the Reynolds number. A threedimensional solid representing the biofilm is then "immersed" in the fluid and coupled to the fluid through a force balance and a velocity matching condition. This approach requires that the material being represented by the immersed solid (i.e., the biofilm) to have a density close to that of the surrounding fluid's density.

The applied body force per unit volume on the fluid, $f$, is related to the force density in the immersed solid, $F$, given by

$$
f(x, t)=\int F(q, r, s, t) \cdot \delta(x-X(q, r, s, t)) \mathrm{d} q \mathrm{~d} r \mathrm{~d} s
$$

where $\delta$ is the three-dimensional Dirac delta function, $x=(x, y, z)$ are the fixed Cartesian coordinates (used for the fluid), $X(q, r, s, t)$ represents the three-dimensional, transient, immersed solid in the three-dimensional Cartesian space, and $q, r$, and $s$ are curvilinear coordinates attached to the material.

The immersed solid force density, $F$, in Equation (3) is the Fréchet derivative of the elastic energy function, $E(X)$, and it is given by:

$$
F=-\frac{\partial E}{\partial X}
$$

There are a number of possible elastic energy functions that describe the connections between the immersed solid points. For example, the points can be connected with rods that resist bending or spring and dashpot combinations (Heys et al., 2008; Kim and Peskin, 2007). Here, the connections between the immersed solid points are modeled as simple, one-dimension springs, and the elastic energy function is typically written as:

$$
E(X)=\frac{1}{2} c_{s} \int\left(\left|\frac{\partial X}{\partial s}\right|-1\right)^{2} \mathrm{~d} s .
$$


The points are not equally spaced, but they are regularly arranged, and each interior point is connected to the six nearest neighbors. It is important to note that even though the solid is modeled using elastic springs, the solid is coupled by force and velocity balances to a viscous fluid occupying the same volume so the biofilm will behave as a viscoelastic material, consistent with experimental measurement of the material properties of biofilms (Klapper et al., 2002; Towler et al., 2003; Vo et al., 2010). Finally, in addition to balancing the forces between the fluid and immersed solid, the velocity of the fluid must be equal to the velocity of the solid. This condition is imposed by:

$$
\frac{\partial X(q, r, s, t)}{\partial t}=\int v(x, t) \cdot \delta(x-X(q, r, s, t)) \mathrm{d} x
$$

Equations (1-6) represent the complete mathematical model for describing fluid-biofilm interaction, and these equations are the same as those solved previously in Vo et al. (2010). All the equations and variables are solved in dimensionless form. The characteristic length scaling is set to the size of the capillary (i.e., the channel height) and the characteristic velocity scaling is set to the peak inflow velocity for the baseline case. The current algorithm does not model biofilm detachment, which was not observed in the experiments, but other biofilm models have been developed that are capable of modeling detachment (e.g., Alpkvist and Klapper, 2007; Bol et al., 2009). The numerical algorithm to solve the model equations has been redesigned, as described below, from that used in Vo et al. (2010) to improve the computational performance and scalability. However, the current algorithm was tested to ensure that it gave the same predictions as the Vo et al. algorithm.

The IBAMR software is used to solve the model equations (Griffith, 2009; Griffith et al., 2007). This software solves the Navier-Stokes and continuity equations [Eqs. (1) and (2)] using the SAMRAI library (Hornung and Kohn, 2002) from Lawrence Livermore National Laboratory, which enables adaptive mesh refinement and MPI-based parallelization. The finite difference discretization of Navier-Stokes equations is fully implicit and based on a staggered grid. The initial fluid grid, before refinement, was coarse (typically $16 \times 16 \times 32$ nodes), but this grid was refined over multiple levels in regions containing the immersed boundary or biofilm points. The refinement was constrained so that the biofilm and the large velocity gradients near the biofilm were entirely within the finest level of refinement. Details of the discretization and refinement criteria are available in Griffith et al. (2007). In a few cases here, the initial coarse grid was refined to $32 \times 32 \times 64$ nodes to ensure that the coarse grid level was acceptable, and no significant difference was observed between the two different coarse grids. Overall, including the immersed boundary, the method is formally second-order, and has been shown to be accurate on a number of standard test problems (Griffith, 2009). The simulations shown in the results section were run on two to eight processors and typically required $2-4 \mathrm{~h}$ to complete. The approximate solution was visualized and analyzed using Visit, also from Lawrence Livermore National Laboratory.

The model domain is a dimensionless $1 \times 1 \times 2$ cuboid; chosen to be a section of a channel used in the MRM experiments described in Hornemann et al. (2009). A noslip boundary condition was set along the top, bottom, and side walls of the domain, and laminar, parabolic flow profile was set along the inlet surface. A zero normal stress and zero tangential velocity boundary conditions were set along the outlet. The shape of the biofilm is based on the shape of experimentally observed biofilms grown in capillary channels and observed microscopically. These biofilm clusters could be described as "hill-shaped" and are mathematically described here using a sine wave. In nature, a large range of biofilm shapes can be observed, but here we are only modeling the relatively young, small biofilm clusters grown in capillary channels by Hornemann et al. The height of the biofilm was varied to simulate different ratios of biofilm height to channel height. The points representing the biofilm (i.e., the solid) were initially spaced to so that the maximum distance between points was approximately onethird the distance between fluid grid points. The result is that the biofilm was essentially impermeable in the rest position (Alpkvist et al., 2006; Mitchell et al., 2009; Peskin, 2002; Stewart, 2003). As the biofilm deforms, some of the solid points are spread apart, and, once the spacing between the solid points approaches the spacing between the fluid grid points, the biofilm becomes slightly permeable (Pintelon et al., 2009).

\section{Results and Discussion}

Every simulation begins with the fluid and biofilm at rest. The initial results are based on a baseline case with a ratio of biofilm height to channel height of 0.15 (close to that of the $0.9 \mathrm{~mm}$ channel experiment by Hornemann et al.) and a Reynolds number of 300 (in the middle of the range examined by Hornemann et al.). Finally, the spring stiffness for the biofilm, $c_{s}$, is set to 0.002 (dimensionless) for the baseline case, which corresponds to a spring stiffness of $6 \times 10^{-5} \mathrm{~g} /\left(\mathrm{mm}^{2} \mathrm{~s}^{2}\right)$ used in the previously validated model. In the simulations, the fluid is accelerated from rest for $1 \mathrm{~s}$, and then held at a steady flow for a total of $10 \mathrm{~s}$. Typically, the biofilm deformation changes for approximately $2-4 \mathrm{~s}$ (depending on the mechanical properties of the biofilm and the Reynolds number), but after the initial transient period, the biofilm deformation reaches a pseudo-steady-state condition where only very small deformation changes persist.

Figure 1a shows the initial biofilm shape when the fluid is at rest, and Figure $1 \mathrm{~b}$ shows the biofilm deformation during the transient phase, $1.16 \mathrm{~s}$ after the left to right flow was initiated. The streamlines in Figure $1 \mathrm{~b}$ show the fluid being deflected upward by the biofilm, thus establishing a 
a

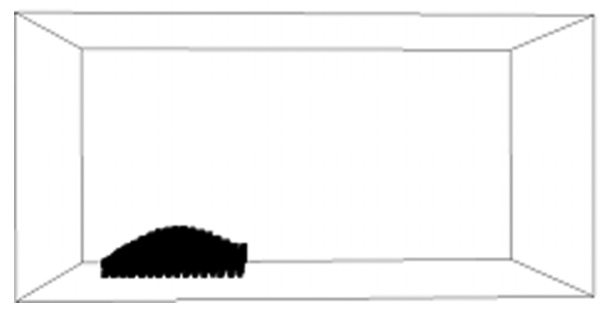

Flow Direction

b

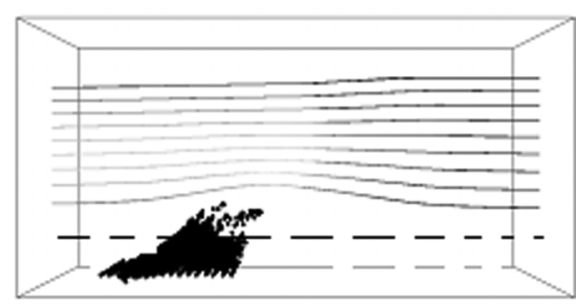

Figure 1. a: The biofilm is at rest, and (b) the biofilm being deformed by the fluid flow from left to right with centerline streamlines shown for the fluid. The particles are stretched apart due to the fluid shear, but they are still connected together with springs (not shown). The biofilm height has increased from the rest position by $20-30 \%$ as indicated by the dashed line.

secondary velocity component, and a small streamer or flap is formed on the downsteam side of the biofilm. Flaps and streamers are commonly observed to form on biofilm clusters in capillary tubes (Taherzadeh et al., 2010), and they can detach after a period of time (a few minutes to a few hours).

A critical observation that can be made by comparing the two biofilm shapes shown in Figure 1 is that the biofilm is not compressed towards the solid surface by fluid shear, as we initially expected, but the maximum biofilm height actually increases slightly (20-30\%) due to the fluid flow. This lift is not caused by a Bernoulli affect (i.e., an increase in velocity causing a pressure minimum immediately above the biofilm) because a pressure minimum is not observed above the biofilm in the model.

Insight into the hydrodynamic forces on the biofilm can be gained from Figure 2, which is a magnified view of the downstream section of the biofilm from Figure $1 \mathrm{~b}$ and shows streamlines that were seeded immediately downstream of the biofilm. In this figure, we can see that there is a dynamic recirculation immediately downstream of the flexible biofilm, and this recirculation generates an upward shear force on the downstream side of the biofilm that causes the points in that section of the biofilm, in particular, to be elevated slightly relative to the rest position. The mathematical modeling results presented thus far, suggest that the unexpectedly large secondary velocity magnitudes measured by Hornemann et al. may be generated by a combination of a downstream recirculation on each biofilm cluster and the lack of stiffness or mechanical rigidity of a biofilm. Obvious questions to examine at this point include: what is the effect of a different biofilm height to channel height ratio? what is the impact of changing the Reynolds number?, and what is the impact of changing the mechanical properties of the biofilm?

Figure 3 summarizes the impact of the ratio of biofilm resting height to channel height on the model prediction of the ratio of maximum secondary velocity to maximum primary velocity. Recalling that for a $2 \mathrm{~mm}$ capillary, which corresponds to a biofilm-to-channel height ratio of approximately 0.05 , Hornemann et al. measured a velocity ratio of $0.12 \pm 0.028$, which is close to the model prediction of 0.09 shown in Figure 3. For a $0.9 \mathrm{~mm}$ capillary (height ratio of approximately 0.11 ), Hornemann et al. measured a velocity ratio of $0.20 \pm 0.003$, and the model once again provides excellent agreement with the experimental result. The agreement is very different for the $0.5 \mathrm{~mm}$ capillary where Hornemann et al. measured a ratio of $0.049 \pm 0.001$, which indicates very small secondary velocities relative to the primary velocity, and the model predicts a velocity ratio
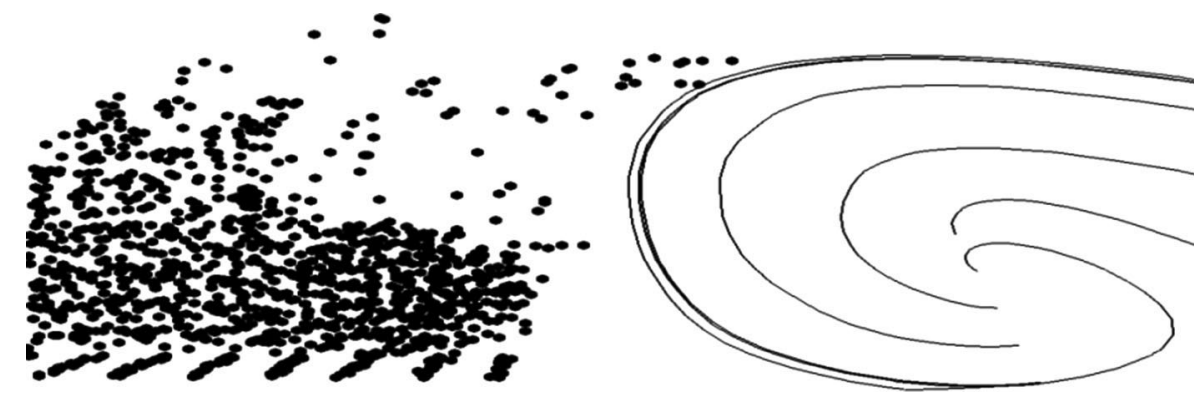

Figure 2. Streamlines were seeded in the flow on the downstream side of the biofilm from Figure $1 \mathrm{~b}$, and they show a recirculation-like pattern in the fluid flow. 


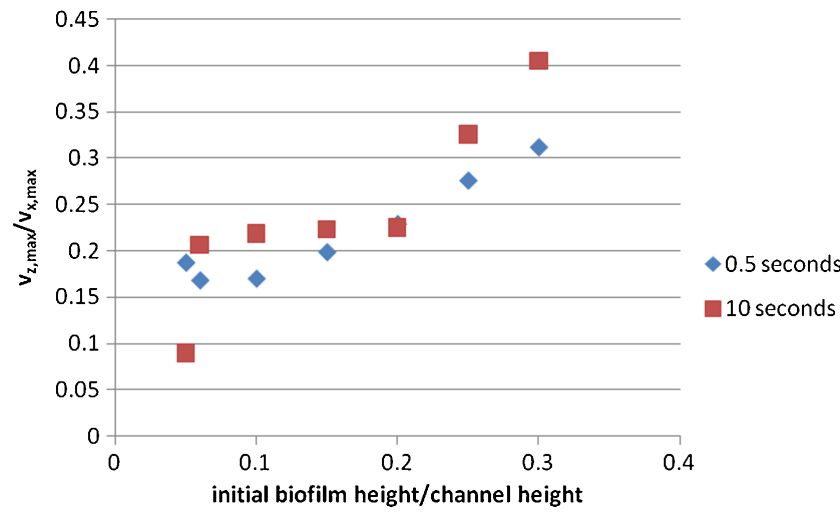

Figure 3. Ratio of $v_{z, \max } / v_{x, \max }$ versus the ratio of initial biofilm height to channel height at two different time points, 0.5 and $10 \mathrm{~s}$, after flow was initiated. [Color figure can be seen in the online version of this article, available at http://wileyonlinelibrary. com/bit]

of 0.23 . We varied the biofilm height slightly in the $0.5 \mathrm{~mm}$ capillary, and the model predictions were consist in showing relatively large velocity ratios on the order of 0.2 . There are at least three possible reasons for this lack of agreement between the model and experimental measurements. First, the smallest capillary had the highest Reynolds number. The impact of flow rate and Reynolds number is examined in the next section, but the results there will indicate that a higher Reynolds number is not a likely explanation for this discrepancy. Second, the geometry of the biofilm has a significant impact on the physics of the fluid-structure interaction, and the biofilms clusters in the $0.5 \mathrm{~mm}$ capillary may have had a significantly different shape from the standard cluster geometry used here. A third possible cause for the discrepancy may be that the biofilm grown in the smallest capillary may have a somewhat different structure than in the larger capillaries. For example, if the biofilm coverage of the capillary wall were more complete in the smallest capillary, it could reduce the effective height of the biofilm. It is impossible to determine exactly what caused the experiment-model discrepancy for the smallest capillary, but the excellent agreement for the two larger capillaries is encouraging.

The second set of parameter variation results show the impact of changing the inflow velocity on the model prediction of maximum secondary velocity to maximum primary velocity (Fig. 4). In the baseline case, the maximum inflow velocity was set to 1.0 (dimensionless). When the inflow velocity is changed to something other than 1.0, the Reynolds number is changed by a proportional amount (i.e., an inflow velocity of 2.0 corresponds to a Reynolds number of 600 instead of 300). We did not change the Reynolds number directly because we wanted to isolate the effects of fluid velocity from other model parameters, including the spring constant, $c_{s}$, density, and viscosity. In general, higher velocities and higher Reynolds number lead to an increase in the ratio of maximum secondary velocity to

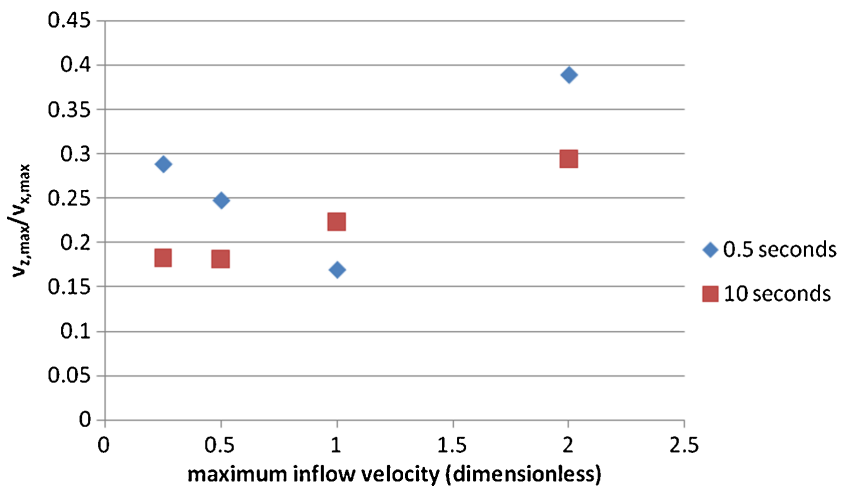

Figure 4. Ratio of $v_{z, \max } / v_{x, \max }$ versus the maximum inflow velocity (dimensionless). An inflow velocity of 1.0 corresponds to a Reynolds number of 300 . Once pseudosteady-state is achieved, $10 \mathrm{~s}$ after the flow is initiated, the secondary-to-primary velocity ratio can be observed to increase with inflow velocity. [Color figure can be seen in the online version of this article, available at http://wileyonlinelibrary.com/bit]

maximum primary velocity. This observation is consistent with the measurements of Hornemann et al. which showed a smaller secondary-to-primary velocity ratio for the lowest Reynolds number flow $(\operatorname{Re} \approx 100)$.

The final set of parameter variation results, shown in Figure 5, describe the impact of changing the mechanical properties of the biofilm (i.e., changing $c_{s}$ in Eq. 6) on the maximum secondary velocity. From these figures, the ratio of maximum secondary velocity to maximum primary velocity reaches a maximum value at a spring stiffness of 0.001 (dimensionless). Before or after the value of 0.001 , the secondary velocity is smaller relative to the primary velocity. In Vo et al. (2010), we showed that spring constants in the range of $3 \times 10^{-5} \mathrm{~g} /\left(\mathrm{mm}^{2} \mathrm{~s}^{2}\right)$ to $6 \times 10^{-5} \mathrm{~g} /\left(\mathrm{mm}^{2} \mathrm{~s}^{2}\right)$ or

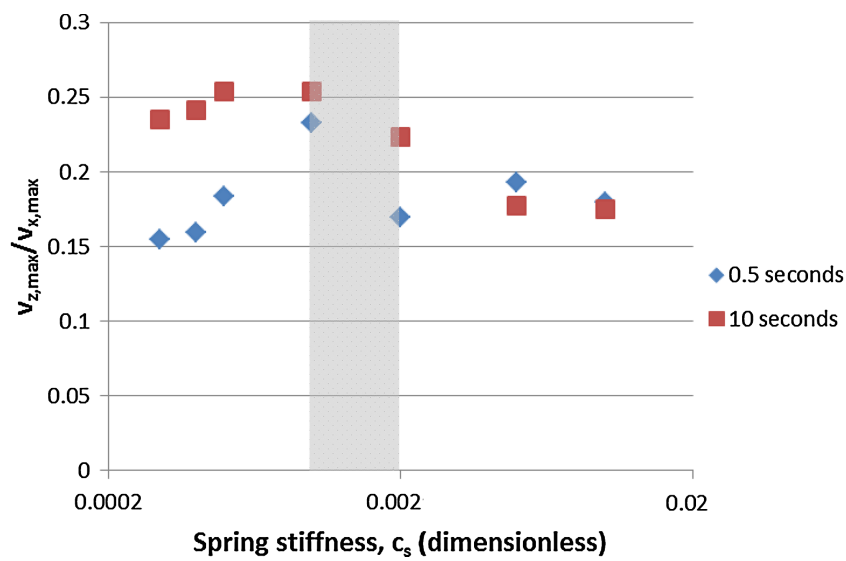

Figure 5. Ratio of $v_{z, \max } / v_{x, \max }$ versus sping stiffness, $c_{s}$ (dimensionless). A higher spring stiffness corresponds to a more rigid biofilm, and a lower spring stiffness represents a softer biofilm. The grey box shows the range of spring stiffness value that resulted in the model agreeing well with experimental measurements (Vo et al., 2010). [Color figure can be seen in the online version of this article, available at http://wileyonlinelibrary.com/bit] 
0.001-0.002 (dimensionless) gave the best agreement with experimental measurements of biofilm deformation. Biofilms modeled as having more compliant mechanical properties (i.e., a spring stiffness $<0.0005$ ) tended to have their upstream regions deflected downward, first, and then the downstream section was also depressed, and a significant recirculation never formed downstream of the biofilm. On the other hand, biofilms modeled as being more rigid (i.e., a spring stiffness $>0.002$ ) tended to resist deformation, and the shear from the downstream recirculation failed to deform the biofilm significantly. This evidence supports the idea that if actual biofilms were significantly softer or stiffer than their observed properties, the nearby secondary velocities would potentially be $20-30 \%$ smaller. It is important to note, however, that the biofilms modeled here are assumed to have homogeneous mechanical properties, but many natural biofilms are known to have heterogeneous properties. The extension of the results here to biofilms with heterogeneous mechanical properties is unknown.

To quantify the impact of secondary velocity on species transport near the biofilm, it is helpful to consider that particles in a flow with a velocity of $1.0 \mathrm{~mm} / \mathrm{s}$ travel past a biofilm cluster of length $100 \mu \mathrm{m}$ in approximately $0.1 \mathrm{~s}$. Given this contact time, $t$, and a self-diffusion coefficient, $D$, for water of $2 \times 10^{-9} \mathrm{~m}^{2} / \mathrm{s}$, a characteristic diffusion distance of $2 \sqrt{D \cdot t}=28 \mu \mathrm{m}$ can be calculated, which implies that only substrate that passes within approximately $28 \mu \mathrm{m}$ of the biofilm can reach the biofilm by diffusion alone for the experimental system studied here. Given this time and a self-diffusion coefficient, $D$, for water of $2 \times 10^{-9} \mathrm{~m}^{2} / \mathrm{s}$, a characteristic diffusion distance of $2 \sqrt{D \cdot t}=28 \mu \mathrm{m}$ can be calculated, which implies that only substrate that passes within approximately $28 \mu \mathrm{m}$ of the biofilm can reach the biofilm by diffusion alone. The Peclet number, $V \cdot L / D$, represents the ratio of convective mass transport to diffusive molecular transport. A characteristic secondary velocity of $0.2 \mathrm{~mm} / \mathrm{s}$ and a characteristic length of $100 \mu \mathrm{m}$ give a Peclet number of 10 , implying that secondary velocities predicted here and observed in Hornemann et al. increase transport of substrates to biofilms by a factor of 10 . A 20-30\% decrease in secondary velocity would subsequently reduce convective mass transport by that same amount, significantly impacting the amount of substrate reaching the biofilm.

\section{Conclusions}

The immersed boundary biofilm model has been previously validated against experimental data, and it was used here to provide insight into the mechanical interaction between a biofilm and fluid flow in a square capillary. The model predicts the formation of a recirculation downstream of a biofilm that lift the biofilm up and enhances (in some cases significantly) the secondary velocities near the biofilm. The magnitude of the secondary velocities relative to the primary velocity is predicted to increase with the ratio of channel height to biofilm height and inflow velocity magnitude. Enhanced secondary velocities should increase the delivery of substrates to the biofilm so it is logical that biofilms would try to maximize these secondary velocities. The mechanical properties of biofilms appear to be tuned to maximize uplift of the biofilm and maximize the generation of secondary velocities over a wide range of flow conditions.

We wish to thank Prof. J. Seymour for helpful comments and suggestions throughout this work. The work was supported by the Flight Attendant Medical Research Institute and NSF grants CMMI-0849433 and DMS-0811275.

\section{References}

Alpkvist E, Klapper I. 2007. Description of mechanical response including detachment using a novel particle model of biofilm/flow interaction. Wat Sci Tech 55:265-273.

Alpkvist E, Picioreanu C, van Loosdrecht MCM, Heyden A. 2006. Threedimensional biofilm model with individual cells and continuum EPS matrix. Biotechnol Bioeng 94(5):961-979.

Bird RB, Stewart WE, Lightfoot EN. 2002. Transport phenomena. New York: John Wiley. xii, p. 895.

Bol M, Mohle RB, Haesner M, Neu TR, Horn H, Krul R. 2009. 3D finite element model of biofilm detachment using real biofilm structures from CLSM data. Biotechnol Bioeng 103(1):177-186.

Chambless JD, Stewart PS. 2007. A three-dimensional computer model analysis of three hypothetical biofilm detachment mechanisms. Biotechnol Bioeng 97(6):1573-1584.

Characklis WG. 1981. Fouling biofilm development-A process analysis. Biotechnol Bioeng 23(9):1923-1960.

Costerton JW, Lewandowski Z, Caldwell DE, Korber DR, Lappin-Scott HM. 1995. Microbial biofilms. Annu Rev Microbiol 49:711-745.

Gjersing EL, Codd SL, Seymour JD, Stewart PS. 2005. Magnetic resonance microscopy analysis of advective transport in a biofilm reactor. Biotechnol Bioeng 89(7):822-834.

Griffith BE. 2009. An accurate and efficient method for the incompressible Navier-Stokes equations using the projection method as a preconditioner. J Comput Phys 228(20):7565-7595.

Griffith BE, Hornung RD, McQueen DM, Peskin CS. 2007. An adaptive, formally second order accurate version of the immersed boundary method. J Comput Phys 223(1):10-49.

Heys JJ, Gedeon T, Knott BC, Kim Y. 2008. Modeling arthropod filiform hair motion using the penalty immersed boundary method. J Biomech 41(5):977-984.

Hornemann JA, Codd SL, Fell RJ, Stewart PS, Seymour JD. 2009. Secondary flow mixing due to biofilm growth in capillaries of varying dimensions. Biotechnol Bioeng 103(2):353-360.

Hornung RD, Kohn SR. 2002. Managing application complexity in the SAMRAI object-oriented framework. Concurrency Comput Pract Exp 14(5):347-368.

Kim Y, Peskin CS. 2007. Penalty immersed boundary method for an elastic boundary with mass. Phys Fluids 19(5):053103-1-053103-18.

Klapper I, Dockery J. 2010. Mathematical description of microbial biofilms. Siam Rev 52(2):221-265.

Klapper I, Rupp CJ, Cargo R, Purvedorj B, Stoodley P. 2002. Viscoelastic fluid description of bacterial biofilm material properties. Biotechnol Bioeng 80(3):289-296.

Lewandowski Z, Beyenal H. 2007. Fundamentals of biofilm research. Boca Raton: CRC Press. 452 p., [2] p. of plates p.

Lewandowski Z, Stoodley P, Altobelli S. 1995. Experimental and conceptual studies on mass-transport in biofilms. Water Sci Technol 31(1):153162 . 
McQueen DM, Peskin CS. 2000. A three-dimensional computer model of the human heart for studying cardiac fluid dynamics. Comput GraphUs 34(1):56-60.

Mitchell AC, Phillips AJ, Hiebert R, Gerlach R, Spangler LH, Cunningham AB. 2009. Biofilm enhanced geologic sequestration of supercritical $\mathrm{CO}_{2}$. Int J Greenhouse Gas Control 3(1):90-99.

Peskin CS. 1977. Numerical-analysis of blood-flow in heart. J Comput Phys 25(3):220-252.

Peskin CS. 2002. The immersed boundary method. Acta Numerica 11:479517.

Picioreanu C, van Loosdrecht MCM, Heijnen JJ. 2000. A theoretical study on the effect of surface roughness on mass transport and transformation in biofilms. Biotechnol Bioeng 68(4):355-369.

Pintelon TRR, von der Schulenburg DAG, Johns ML. 2009. Towards optimum permeability reduction in porous media using biofilm growth simulations. Biotechnol Bioeng 103(4):767-779.

Rani SA, Pitts B, Stewart PS. 2005. Rapid diffusion of fluorescent tracers into Staphylococcus epidermidis biofilms visualized by time lapse microscopy. Antimicrob Agents Chemother 49(2):728-732.

Stewart PS. 2003. Diffusion in biofilms. J Bacteriol 185(5):1485-1491.
Stewart PS, Rani SA, Gjersing E, Codd SL, Zheng Z, Pitts B. 2007. Observations of cell cluster hollowing in Staphylococcus epidermidis biofilms. Lett Appl Microbiol 44(4):454-457.

Taherzadeh D, Picioreanu C, Kuttler U, Simone A, Wall WA, Horn H. 2010. Computational study of the drag and oscillatory movement of biofilm streamers in fast flows. Biotechnol Bioeng 105(3):600610.

Towler BW, Rupp CJ, Cunningham AB, Stoodley P. 2003. Viscoelastic properties of a mixed culture biofilm from rheometer creep analysis. Biofouling 19(5):279-285.

Vo GD, Brindle E, Heys J. 2010. An experimentally validated immersed boundary model of fluid-biofilm interaction. Water Sci Technol 61(12):3033-3040.

Wagner M, Manz B, Volke F, Neu TR, Horn H. 2010. Online assessment of biofilm development, sloughing and forced detachment in tube reactor by means of magnetic resonance microscopy. Biotechnol Bioeng 107(1):172-181.

Zhu LD, Peskin CS. 2002. Simulation of a flapping flexible filament in a flowing soap film by the immersed boundary method. J Comput Phys 179(2):452-468. 